\title{
First Clinical Report on Comparative Treatment and Survival Outcomes in Second Cancers after Primary Head and Neck Cancer: A Population-Based Study
}

Xin Wang ${ }^{1}$, Elizabeth A. Mauer ${ }^{2}$, Paul Christos ${ }^{2}$, Julia Manzerova ${ }^{1}$, A. Gabriella Wernicke ${ }^{1}$, Bhupesh Parashar ${ }^{3}$

1. Stich Radiation Oncology, NewYork-Presbyterian/Weill Cornell Medical Center 2. Division of Biostatistics and Epidemiology, Department of Healthcare Policy and Research, New YorkPresbyterian/Weill Cornell Medical Center 3. Radiation Oncology, NewYork-Presbyterian/Weill Cornell Medical Center

$\square$ Corresponding author: Bhupesh Parashar, bup9001@med.cornell.edu Disclosures can be found in Additional Information at the end of the article

\section{Abstract}

\section{Introduction}

To compare patients' survival of second primary malignancy (SPM) after head and neck squamous cell carcinoma (HNSCC).

\section{Methods}

The Surveillance, Epidemiology, and End Results (SEER) database was utilized (1973-2011). The Kaplan-Meier method with log-rank test was used to compare the overall survival (OS) and cause-specific survival (CSS) among treatment methods from the time of diagnosis of SPMs. Cox proportional regression models were used to adjust the impact for risk factors on CSS.

\section{Results}

A total of 3,038 patients were identified (5-yr OS 22.6\% (21.0-24.3\%)). For head and neck (HN) SPMs, the patients who received 'conservative surgery with radiation' had the best 5-yr OS (65.2\% (48.9-86.9\%)); and the 'conservative surgery' group had the best 5-yr CSS (89.9\% (85.694.5\%)). For lung SPMs, the 'radical surgery' group showed the best survival (2-yr OS 60.8\% (56.0-66.1\%), 2-yr CSS 70.6\% (65.8-75.8\%), respectively). Esophagus SPMs had poor prognosis, with no difference among the treatment groups. In lung SPMs, younger age $(p<0.001)$ and black race $(p<0.05)$ were most favorable CSS predictors.

Received 04/11/2017

Review began 04/14/2017 Review ended 05/20/2017 Published 05/29/2017

\section{C) Copyright 2017}

Wang et al. This is an open access article distributed under the terms of the Creative Commons Attribution License CC-BY 3.0., which permits unrestricted use, distribution, and reproduction in any medium, provided the original author and source are credited.

\section{Conclusions}

The prognosis of SPMs after HNSCC is worse compared with corresponding primary tumor. Conservative surgery with or without radiation showed the most favorable outcomes in HN SPMs.

Categories: Radiation Oncology, Miscellaneous, Oncology

Keywords: seer, head and neck cancer, second primary malignancy, treatment types, survival 


\section{Introduction}

Head and neck (HN) cancer patients are known to be at significantly elevated risk of second primary malignancies (SPMs), compared with the matched population and SPMs are the leading cause of mortality (30\% [1-2]) among long-term head and neck squamous cell carcinoma (HNSCC) survivors. Effective and successful SPM treatment is therefore a crucial part of longterm HNSCC management.

The concept of 'field cancerization', which was first introduced by Slaughter, et al. [3] in 1953, has been used to explain the high occurrence of SPMs in HNSCC. The classic view of 'field cancerization' is that exposure of environmental carcinogens, such as tobacco and alcohol use, may induce premalignant disease in large mucosal area and elevate the secondary cancer risk throughout the aero-digestive tract. Additional studies have further confirmed that some SPMs share a similar genetic pattern with the index tumor [4]. Other risk factors, such as aging and human papillomavirus (HPV)-seronegative status, etc. have been proven to significantly increase the incidence of SPMs [5-6]. The most common site of HNSCC SPMs is lung, followed by HN, and esophagus [5, 7-8], attributing to about $60 \%$ of all SPMs combined.

Patients who develop an SPM tend to have a significantly worse survival, compared to those who do not [7]. Head and neck SPMs have a relatively better prognosis than SPMs arising in lung or esophagus, with a 5 -yr survival of $61 \%, 19 \%$, and $0 \%$, respectively [2, 9]. Lung SPM patients after an HNSCC primary tumor have a worse prognosis compared with the matched general population with primary lung cancer, regardless of the histology [10]. All these findings highlight the importance of better understanding and management of HNSCC SPMs.

In this population-based study, we aim to compare the survival outcomes across treatment types in HNSCC patients who developed an HN, lung, or esophagus SPM and to determine whether there is any association between risk factors and the survival in SPM patients.

\section{Materials And Methods}

\section{The Surveillance, Epidemiology, and End Results program and Multiple Primary-Standardized Incidence Ratios session}

The Surveillance, Epidemiology, and End Results (SEER) program of the National Cancer Institute (NCI) is an authoritative source of information on cancer incidence and survival in the US, which routinely collects data on patient demographics, tumor site, morphology, stage at diagnosis, course of treatment, and follow-up for vital status, with quality control routinely performed since 1973 and now covers approximately $28 \%$ of the US population [11]. The NCI does not require institutional board approval for the use of SEER data.

The Standardized Incidence Ratios' (MP-SIR) session is a session under the SEER program, in which a defined cohort of people previously diagnosed with cancer is followed through time to compare their subsequent primary cancer experience to the matching general population [12]. By extracting the detailed information of the secondary cancers, one can also look into the risk factors, management techniques, and survival outcomes of specific SPMs [13].

\section{Study population and treatment categories}

The SEER*Stat 8.2.1 software package (SEER, National Cancer Institute, MD, USA) and the MPSIR session were used to identify patients who were diagnosed with a primary HNSCC and then developed SPMs between 1973 and 2011 in the nine registries of the SEER program. Patients with HNSCC were defined using the International Classification of Diseases for Oncology, third edition (ICD-O-3), histology codes for squamous cell carcinoma (8070-8078), and subsite codes 
for head and neck cancer (oral cavity, oropharynx, hypopharynx, larynx, and nasopharynx). All patients included were in 'active follow-up' or 'originally inactive, then active follow-up' as defined in the database. Given the relatively small study population for each SPM subsite, the stage at presentation was categorized according to the SEER historic stage A codes as 'localized' (localized without lymph node involvement or distant metastases, NOM0), 'regional' (locally advanced or lymph node-positive without distant metastases, $\mathrm{N}+\mathrm{M} 0$ ), or 'distant' (distant metastases, any N M1) [14]. SPM was defined as an invasive solid tumor developing $\geqslant 2$ months after an index HNSCC as per National Cancer Institute criteria [15]. Patients with HN, lung, and esophagus SPMs were drawn from the case listing sheet, and the ones who died from the index tumor, developed a third malignancy, or had an un-staged disease were excluded.

Two SEER variables 'Site specific surgery (1983-1997)' and 'Surgery of the primary site (1988+)' were recoded together to define and categorize the surgical treatment received. Radiation therapy (RT) was limited to 'Beam radiation' (SEER code 1) and 'Combination of beam and implants or isotopes' (SEER code 4). For HN SPMs, treatment categories were defined as: 'conservative surgery (CS, SEER codes 10-30, including electroautery, cryosurgery, laser surgery, local excision, partial pharyngectomy, tonsillectomy, and partial laryngectomy, etc., but excluded excisional biopsy)', 'radical surgery (RS, SEER codes 40-90, including radical excision with/without lymph nodes dissection, total laryngectomy, and pharynolaryngectomy, etc.)', 'radiation (RT)', 'conservative surgery with radiation (CSRT)', 'radical surgery with radiation (RSRT)', and 'no treatment' group. We acknowledge that for pharynx, in 'Surgery of the primary site (1998+)' manual, code 30 also includes total pharyngectomy. However, for simplicity, we categorized it in the CS group. CS and CSRT groups (including local tumor destruction as laser ablation, cryosurgery, electroautery, and photodynamic therapy with/without radiation) were excluded from lung and esophagus SPMs because of atypical treatment patterns and very small patient number. For lung SPMs, RS was defined as SEER codes 20-90, included wedge resection, segmental resection, lobectomy, pneumonectomy, radical pneumonectomy, and extended pneumonectomy. For esophagus SPMs, RS was defined as SEER codes 40-90 in the 'Site specific surgery (1983-1997)' manual and 30-90 in the 'Surgery of the primary site (1998+)' manual and included partial/total esophagectomy with/without laryngectomy and/or gastrectomy.

\section{Statistical analysis}

Descriptive statistics were calculated to characterize the study cohort in relation to demographic factors, social-economic status, and tumor characteristics. The primary endpoint was cancer-specific survival (CSS). For CSS, the patients with deaths due to causes other than cancer were censored. A secondary endpoint was overall survival (OS). Time for both CSS and OS was calculated as time in months from the diagnosis of the SPM tumor to the date of death from any cause, or to the date of last follow-up if a patient did not have a recorded death. Kaplan-Meier survival analyses were performed to evaluate CSS and OS by SPM tumor staging and treatment group, stratified by SPM subsite. All analyses were used to produce relevant survival estimates and to employ the log-rank test for comparison of CSS/OS between the different groups of interest.

Unadjusted Cox proportional hazards regression analyses were performed to evaluate the impact of all demographic, social-economic, and tumor factors on CSS, stratified by SPM subsite. Multivariable Cox proportional hazards regression analyses was then performed with all variables included that were significant at the 0.20 alpha level from the unadjusted analyses. 'Age at index tumor' and 'year of index tumor' were excluded because of their collinearity with corresponding SPM data. Competing-risks survival regression was also performed to check the multivariable CSS hazard ratios by accounting for the competing event of death due to causes other than cancer (based on Fine and Gray's proportional subhazards model). All p-values are two-sided with statistical significance evaluated at the 0.05 alpha level. Ninety-five percent 


\section{Cureus}

confidence intervals (CI) for unadjusted and adjusted HRs and subhazard ratios were calculated to assess the precision of the obtained estimates. All analyses were performed using R-3.2.0 for Windows 64 bit (R-statistics.com, Vienna, Austria).

\section{Results}

\section{Description of study population}

A total of 3,138 patients with a median follow-up of 12 months were identified, of which, 1,056 patients developed an HN SPM (33.7\%), 1,717 patients developed a lung SPM (54.7\%), and 265 patients developed an esophagus SPM (8.4\%). The patient and tumor characteristics are summarized in Table 1 . The median age for index and secondary tumor was 61 years, and 69 years, respectively. The median time interval from the diagnosis of an index tumor to an SPM was 70 months (range 2-242 months). It was found that $51.5 \%$ had a localized or regional (42.7\%) index tumor. In patients with lung SPMs, $45.1 \%$ of patients $(\mathrm{N}=775)$ had distant disease at diagnosis (Table 1).

\section{Patients by Subsite}

\begin{tabular}{|c|c|c|c|c|}
\hline & All Sites & HN & Lung & Esophagus \\
\hline & N (\%) & N (\%) & N (\%) & N (\%) \\
\hline Characteristic & $\mathrm{N}=3038$ & $\mathrm{~N}=1056$ & $\mathrm{~N}=1717$ & $\mathrm{~N}=265$ \\
\hline Median Follow-up Time (mo) & 12 & 30 & 8 & 8 \\
\hline \multicolumn{5}{|l|}{ Gender } \\
\hline Male & 2221 (73.1) & 763 (72.3) & 1244 (72.5) & $214(80.8)$ \\
\hline Female & 817 (26.9) & $293(27.8)$ & $473(27.5)$ & 51 (19.3) \\
\hline
\end{tabular}

Ethnicity

White

Black

Other

Age of Index Tumor (yo)

$\leq 45$

46-55

$56-65$

$66-75$

$\square 75$

Age of SPM (yo)

$\leq 45$

46-55

$\begin{array}{llll}2625(86.4) & 943(89.3) & 1497(87.2) & 185(69.8) \\ 289(9.5) & 75(7.1) & 155(9.0) & 59(22.3) \\ 124(4.1) & 38(3.6) & 65(3.8) & 21(7.9)\end{array}$

$\begin{array}{llll}216(7.1) & 119(11.3) & 82(4.8) & 15(5.7) \\ 711(23.4) & 258(24.4) & 377(22.0) & 76(28.7) \\ 1161(38.2) & 354(33.5) & 704(41.0) & 103(38.9) \\ 743(24.5) & 241(22.8) & 443(25.8) & 59(22.3) \\ 207(6.8) & 84(8.0) & 111(6.5) & 12(4.5)\end{array}$

$275(9.1) \quad 119(11.3)$

$12(0.7)$

5 (1.9)

125 (7.3) $31(11.7)$ 


\section{Cureus}

56-65

$66-75$

$\square 75$

Year at diagnosis of Index Tumor

1973-1980

1981-1990

1991-2000

2001-2011

Year at diagnosis of SPM

1973-1980

1981-1990

1991-2000

2001-2011

Median Months since Index Tumor (mo)

\section{Marital Status}

Unmarried

Married

Unknown

\% At Least Bachelors Degree (2000)

$\leq 25$

$\square 25$

Median Family Income (in Ks, 2000)

$\leq 50$

$\square 50$

949 (31.2)

2089 (68.8)

\% Families below Poverty (2000)

$>=10$

$<10$

Index Tumor Staging

Localized

1564 (51.5)

Regional

718 (23.6)

$573(18.9)$

1126 (37.1)

1382 (45.5)

70.00

1176 (38.7)

1187 (39.1)

1851 (60.9)

599 (19.7)

2439 (80.3)

1297 (42.7)
866 (28.5)

$317(30.0)$

$465(27.1)$

$84(31.7)$

1125 (37.0)

$327(31.0)$

$699(40.7)$

$99(37.4)$

$256(24.2)$

$416(24.2)$

$46(17.4)$

408 (13.4) $186(17.6)$

$170(9.9)$

$52(19.6)$

1015 (33.4) 319 (30.2)

$580(33.8)$

$116(43.8)$

$1042(34.3) \quad 333(31.5)$

$648(37.7)$

$61(23.0)$

$218(20.6)$

319 (18.6)

36 (13.6)

$56(1.8) \quad 45$ (4.3)

474 (15.6) 208 (19.7)

$296(28.0)$

$507(48.0)$

73.00

N/A

$11(4.2)$

$372(35.2)$

$606(57.4)$

78 (7.4)

$197(11.5)$

$69(26.0)$

$723(42.1) \quad 107(40.4)$

797 (46.4) $\quad 78(29.4)$

68.00

67.00

397 (37.6) 701 (40.8)

89 (33.6)

$659(62.4) \quad 1016(59.2) \quad 176(66.4)$

$328(19.1)$

$76(28.7)$

$861(81.5) \quad 1389(80.9)$

$189(71.3)$
$645(61.1) \quad 813(47.4)$

$106(40.0)$

$359(34.0) \quad 795(46.3) \quad 143(54.0)$ 


\section{Cureus}

Distant

SPM Tumor Staging
1136 (37.4)

Regional

Distant

Treatment

CS

RS

RT

CSRT

RSRT

None

Vital Status

Alive

Noncancer Deaths

SPM Specific Deaths

Unknown Cause Deaths
981 (32.3)

$177(5.8)$

921 (30.3)
$52(4.9)$

109 (6.3)

$16(6.0)$
$270(25.6)$

307 (29.1) $384(22.4)$

$163(15.4) \quad 623(36.3)$

$45(4.3)$

$83(7.9)$

$188(17.8)$

$85(5.0)$

$625(36.4)$

$56(21.1)$

N/A

50 (18.9)

N/A

$36(13.6)$
$123(46.4)$

$403(38.2) \quad 202(11.8) \quad 25(9.4)$

$360(34.1) \quad 289(16.8) \quad 36(13.6)$

$209(19.8) \quad 1168(68.0) \quad 195(73.6)$

$84(8.0) \quad 58(3.4)$

9 (3.4)

\section{TABLE 1: Descriptive Characteristics by SPM Subsite (1973-2011)}

Abbreviations: CS, conservative surgery; CSRT, conservative surgery with radiation; HN, head and neck; mo, months; RS, radical surgery; RSRT, radical surgery with radiation; RT, radiation therapy; SPM, second primary cancer; yo, years.

\section{Overview of SPM survival by subsite}

The study cohort had a median OS of 14 months (13-15 months) and a median CSS of 22 months (20-26 months). HN SPMs (Table 2) 5-yr OS was 44.0\%, (40.7-47.5\%) and 5-yr CSS 75.6\%, (72.4-78.9\%). Patients with esophagus SPM had a 5-yr OS and 5-yr CSS less than 10.0\%. The SPM staging was closely correlated with survival, except esophageal SPMs where there was no survival difference between localized versus non-localized SPMs (Figures $1 \mathrm{~A}-1 \mathrm{C}$ ). 


\section{Cureus}

\begin{tabular}{|c|c|c|c|c|c|}
\hline \multirow[t]{2}{*}{ Site } & $5-y r$ OS & $95 \% \mathrm{Cl}$ & 5-yr CSS & $95 \% \mathrm{Cl}$ & HR (CSS) \\
\hline & (\%) & (\%) & (\%) & (\%) & \\
\hline All Sites & 22.6 & $21.0-24.3$ & 38.8 & $36.8-40.9$ & NA \\
\hline HN (R) & 44.0 & $40.7-47.5$ & 75.6 & 72.4-78.9 & 1.000 \\
\hline Lung & 12.4 & $10.8-14.2$ & 20.5 & $18.3-23.0$ & $6.152^{\star \star \star}$ \\
\hline Esophagus & 4.9 & $2.7-8.9$ & 8.7 & $5.1-14.8$ & $7.051^{\star \star \star}$ \\
\hline
\end{tabular}

\section{TABLE 2: Overview of Survival Outcomes by Subsite}

Abbreviations: $\mathrm{Cl}$, confidence interval; CSS; cause-specific survival; HN, head and neck; HR, hazard ratio; OS, overall survival; R, reference.

${ }^{* \star *} p<0.001$

A

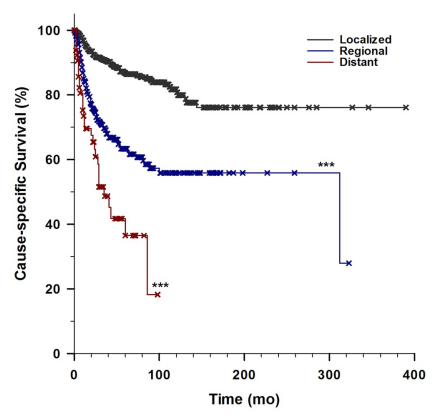

B

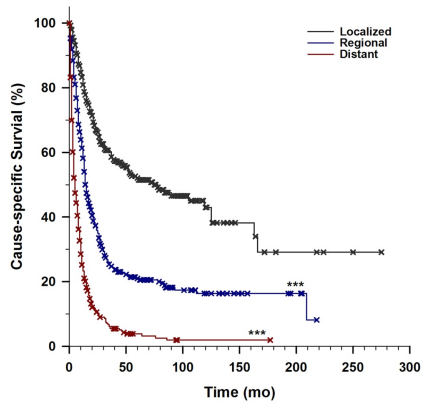

C

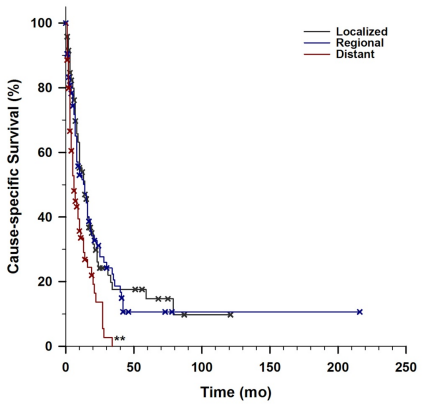

FIGURE 1: Cause-specific Survival by Stage

A. Cause-specific survival of HN SPMs by SPM Staging (Log-rank <0.001)

B. Cause-specific survival of lung SPMs by SPM Staging (Log-rank <0.001)

C. Cause-specific survival of esophagus SPMs by SPM Staging (Log-rank<0.001)

${ }^{\star \star} \mathrm{p}<0.01,{ }^{\star \star \star} \mathrm{p}<0.001$ compared with corresponding localized SPMs

\section{Correlation between treatment type and site-specific survival}

Head and Neck

For HN SPMs, surgical resection was the most frequently performed treatment (RS 29.1\% and CS 25.6\%, Table 1). Patients who received CSRT had 2-yr OS of 87.1\% (77.1-98.4\%) and a 5-yr OS 65.2\% (48.9-86.9\%), while patients who received CS showed a 5-yr CSS of 89.9\% (85.6-94.5\%). The RSRT group had 5-yr CSS of 57.9\% (46.2-72.6\%) (Table 3). Interestingly, the 'no treatment' 


\section{Cureus}

group revealed a comparable survival to RS (5-yr CSS 74.5\% (67.6-82.1\%)), especially in localized SPMs (unadjusted HR of 0.548 (p<0.1)) (Table 4).

\begin{tabular}{|c|c|c|c|c|c|c|}
\hline \multirow{2}{*}{ SPM Site } & Treatment & 2-yr OS (95\% Cl) & 5-yr OS (95\% Cl) & 2-yr CSS (95\% Cl) & 5-yr CSS (95\% Cl) & HR \\
\hline & & (\%) & (\%) & (\%) & (\%) & cSs \\
\hline \multirow{6}{*}{ HN } & CS & 78.3 (73.2-83.8) & $57.2(50.7-64.6)$ & 93.0 (89.6-96.5) & $89.9(85.6-94.5)$ & $0.295^{\star \star \star}$ \\
\hline & RS (R) & $61.3(55.9-67.1)$ & $35.3(29.9-41.6)$ & $84.5(80.0-89.2)$ & $72.1(65.8-78.9)$ & 1.000 \\
\hline & RT & $60.0(52.6-68.4)$ & $35.9(28.3-45.4)$ & $72.0(64.8-80.0)$ & $67.4(59.5-76.3)$ & 1.375 \\
\hline & CSRT & $87.1(77.1-98.4)$ & $65.2(48.9-86.9)$ & $97.5(92.8-100.0)$ & $82.1(66.7-100.0)$ & $0.364^{*}$ \\
\hline & RSRT & $62.4(52.4-74.2)$ & 36.0 (26.3-49.2) & $81.4(72.6-91.1)$ & $57.9(46.2-72.6)$ & 1.484 . \\
\hline & None & $63.8(56.9-71.4)$ & $47.7(40.4-56.3)$ & $79.9(73.8-86.5)$ & $74.5(67.6-82.1)$ & 1.017 \\
\hline \multirow{6}{*}{ Lung } & Treatment & 1-yr OS (95\% Cl) & 2-yr OS (95\% Cl) & 1-yr CSS $(95 \%$ Cl) & 2-yr CSS (95\% Cl) & HR \\
\hline & & (\%) & (\%) & (\%) & (\%) & CSS \\
\hline & RS (R) & $73.2(68.9-77.9)$ & $60.8(56.0-66.1)$ & $81.3(77.3-85.5)$ & $70.6(65.8-75.8)$ & 1.000 \\
\hline & RT & $35.1(31.5-39.2)$ & 15.0 (12.3-18.2) & 40.4 (36.5-44.7) & $22.0(18.5-26.0)$ & $3.765^{\star \star \star}$ \\
\hline & RSRT & $75.6(66.8-85.5)$ & $36.3(27.1-48.6)$ & 78.9 (70.4-88.3) & $39.2(29.5-52.1)$ & $1.959^{\star \star \star}$ \\
\hline & None & $16.4(13.6-19.7)$ & 7.1 (5.26-9.65) & 21,0 (17.8-24.9) & $11.6(8.92-15.1)$ & $6.542^{\star \star \star}$ \\
\hline \multirow{6}{*}{ Esophagus } & Treatment & 1-yr OS (95\% Cl) & 2-yr OS (95\% Cl) & 1-yr CSS (95\% Cl) & 2-yr CSS (95\% Cl) & HR \\
\hline & & (\%) & (\%) & (\%) & (\%) & cSs \\
\hline & RS (R) & $51.9(39.7-67.8)$ & NA & $61.4(48.8-77.3)$ & NA & 1.000 \\
\hline & RT & $36.9(29.1-46.8)$ & NA & $44.0(35.5-54.5)$ & NA & 1.277 \\
\hline & RSRT & $35.5(22.5-55.9)$ & NA & $47.8(32.7-69.9)$ & NA & 1.080 \\
\hline & None & $26.1(16.7-40.9)$ & NA & 29.4 (19.1-45.3) & NA & $2.019^{\star \star}$ \\
\hline \multicolumn{7}{|c|}{ TABLE 3: Survival of SPMs by Subsite } \\
\hline \multicolumn{7}{|c|}{$\begin{array}{l}\text { Abbreviations: SPM, second primary cancer; CS, conservative surgery; RS, radical surgery; RT, radiation therapy; CSRT, } \\
\text { conservative surgery with radiation; RSRT, radical surgery with radiation; HN, head and neck; R, reference; OS, overall survival; } \\
\text { CSS; cause-specific survival; HR, hazard ratio; Cl: confidence interval. }\end{array}$} \\
\hline \multicolumn{7}{|c|}{$. p<0.1,{ }^{*} p<0.05,{ }^{* *} p<$} \\
\hline
\end{tabular}




\section{Cureus}

\begin{tabular}{|c|c|c|c|c|c|c|c|}
\hline \multirow{3}{*}{ SMP Site } & \multirow{2}{*}{ Treatment } & \multicolumn{3}{|l|}{ Localized } & \multicolumn{3}{|l|}{ Regional } \\
\hline & & 5-yr CCS & $95 \% \mathrm{Cl}$ & HR & 5-yr CSS & $95 \% \mathrm{Cl}$ & HR \\
\hline & & (\%) & & css & (\%) & & cSS \\
\hline \multirow{6}{*}{ HN } & CS & 92.0 & $87.8-96.4$ & $0.339^{\star \star \star}$ & 82.8 & $65.3-100.0$ & $0.304^{\star}$ \\
\hline & RS (R) & 82.7 & $75.5-90.7$ & 1.000 & 62.6 & 52.3-74.8 & 1.000 \\
\hline & RT & 79.7 & 68.9-92.2 & 0.982 & 61.3 & $50.7-74.1$ & 1.497 \\
\hline & CSRT & 80.0 & $57.4-100.0$ & 0.523 & 83.0 & $63.5-100.0$ & 0.346 \\
\hline & RSRT & 75.0 & $50.3-100.0$ & 0.952 & 60.6 & $47.0-78.1$ & 1.170 \\
\hline & None & 88.0 & $81.8-94.7$ & 0.548 . & 47.4 & 31.3-71.6 & $2.671^{\star \star}$ \\
\hline \multirow{6}{*}{ Lung } & Treatment & 2-yr CSS & $95 \% \mathrm{Cl}$ & HR & 2-yr CSS & $95 \% \mathrm{Cl}$ & HR \\
\hline & & (\%) & & css & (\%) & & css \\
\hline & RS (R) & 78.5 & $72.9-84.4$ & 1.000 & 62.0 & 53.7-71.6 & 1.000 \\
\hline & $\mathrm{RT}$ & 47.4 & $37.6-59.7$ & $2.619^{\star \star \star \star}$ & 26.0 & 20.3-33.4 & $2.625^{\star \star}$ \\
\hline & RSRT & 75.0 & $54.1-100.0$ & 1.586 & 35.6 & $24.7-51.3$ & $1.500^{*}$ \\
\hline & None & 36.6 & $25.3-52.7$ & $4.538^{\star \star \star}$ & 12.8 & 7.1-23.1 & $5.210^{* \star *}$ \\
\hline \multirow{6}{*}{ Esophagus } & Treatment & 2-yr CSS & $95 \% \mathrm{Cl}$ & HR & 2-yr CSS & $95 \% \mathrm{Cl}$ & HR \\
\hline & & (\%) & & CSS & $(\%)$ & & css \\
\hline & RS (R) & NA & NA & 1.000 & NA & & 1.000 \\
\hline & ${ }_{R T}$ & NA & NA & 1.674 & NA & & 0.959 \\
\hline & RSRT & NA & NA & 1.407 & NA & & 0.818 \\
\hline & None & NA & NA & 2.166 . & NA & & 1.502 \\
\hline
\end{tabular}

\section{TABLE 4: Cause Specific Survival of SPMs by Subsite and Stage}

Abbreviations: SPM, second primary cancer; CS, conservative surgery; RS, radical surgery; RT, radiation therapy; CSRT, conservative surgery with radiation; RSRT, radical surgery with radiation; HN, head and neck; R, reference; OS, overall survival; CSS; cause-specific survival; HR, hazard ratio; CI: confidence interval.

$. p<0.1,{ }^{*} p<0.05,{ }^{* *} p<0.01,{ }^{\star * *} p<0.001$

Lung

In the lung SPM cohort, 591 patients (83.5\%) were diagnosed with a regional or distant disease and radiation was the most commonly used treatment. It was found that 384 cases (22.4\%) (232 localized, 60.4\%) received surgical resection as the only treatment (Table 1). The RS group had a 


\section{Cureus}

2 -yr OS of 60.8\% (56.0-66.1) and 2-yr CSS of 70.6\% (65.8-75.8\%), followed by the RSRT group (Table 3), which remained to be the most superior when categorized into stages (Table 4).

\section{Esophagus}

In the esophagus SPM group, (Tables 2-3), 1-yr OS was 51.9\%, (39.7-67.8\%) and 1-yr CSS was 61.4\%, (48.8-77.3\%), which was observed in RS group (Table 3).

\section{Impact on survival}

In the multivariable model (Table 5), 'SPM staging' was an independent predictor of CSS among all three subsites. 'Index tumor staging' was also adversely correlated with survival except for esophagus SPMs. Younger age at diagnosis and being black were correlated with better survival outcome in lung SPM patients.

\begin{tabular}{|c|c|c|c|c|}
\hline \multirow[b]{2}{*}{ Factors } & \multicolumn{2}{|l|}{ HN } & \multicolumn{2}{|l|}{ Lung } \\
\hline & HR & $95 \% \mathrm{Cl}$ & HR & $95 \% \mathrm{Cl}$ \\
\hline Age of SPM & 1.012 & $0.999-1.026$ & $1.011^{\star \star}$ & $1.005-1.018$ \\
\hline Months since Index Tumor & & & 1.000 & $0.999-1.001$ \\
\hline \multicolumn{5}{|l|}{ Gender } \\
\hline Male (referent) & 1.000 & & 1.000 & \\
\hline Female & 0.760 & $0.540-1.072$ & 0.926 & $0.810-1.057$ \\
\hline \multicolumn{5}{|l|}{ Ethnicity } \\
\hline White (R) & 1.000 & & 1.000 & \\
\hline Black & 1.226 & $0.736-2.042$ & $0.783^{\star}$ & $0.629-0.975$ \\
\hline Other & 0.763 & $0.333-1.749$ & 0.982 & $0.722-1.337$ \\
\hline
\end{tabular}

\section{Marital Status}

Unmarried (R)

1.000

1.000

Married

0.827

$0.609-1.124$

0.914

$0.808-1.033$

0.767 .

$0.560-1.031$

Unknown

0.964

$0.535-1.735$

0.966

$0.658-1.417$

1.005

$0.430-2.349$

\% At Least Bachelors Degree

$\leq 25(R)$

$\square 25$

\section{Median Family Income (K)}

$\leq 50(\mathrm{R})$

$\square 50$

\% Families below Poverty
1.000

1.144

$0.757-1.730$

\section{Esophagus}




\section{Cureus}

$>=10(R)$

$<10$

Index Tumor Staging

Localized (R)

1.000

Regional

Distant

SPM Tumor Staging

Localized (R)

1.000

Regional

Distant

Treatment

CS

$\mathrm{RS}(\mathrm{R})$

RT

CSRT

RSRT

None

1.233

1.027

1.385
1.000

0.797

$0.507-1.254$

$1.569^{* *} \quad 1.168-2.107$

$1.186^{\star \star} \quad 1.048-1.341 \quad 1.122$

0.834-1.509

$2.180^{* *} \quad 1.252-3.793$

1.171

$0.904-1.518$

0.626

$0.325-1.205$

1.000

1.000

$2.804^{\star * \star} \quad 2.009-3.914$

$1.985^{\star \star \star} \quad 1.642-2.400$

1.127

0.795-1.598

$5.247^{\star \star \star} \quad 3.384-8.138$

$3.427^{\star \star *} \quad 2.826-4.156$

$1.714^{\star *}$

$1.182-2.484$

$0.429^{\star *} \quad 0.257-0.715$

$\begin{array}{ll}1.000 & 1.000\end{array}$

1.000

$0.842-1.807$

$2.426^{\star \star *} \quad 1.976-2.978$

1.200

0.802-1.795

$0.430 \quad 0.156-1.189$

NA

NA

0.656-1.607

$1.469^{*}$

1.073-2.012

1.013

0.598-1.718

0.924-2.076

$3.475^{\star \star \star} \quad 2.793-4.323$

$1.624^{\star}$

1.010-2.612

\section{TABLE 5: Multivariable Analysis of Impact of Factors on CSS by Subsite}

Abbreviations: SPM, second primary cancer; CS, conservative surgery; RS, radical surgery; RT, radiation therapy; CSRT, conservative surgery with radiation; RSRT, radical surgery with radiation; HN, head and neck; R, reference; CSS; cause-specific survival; HR, hazard ratio; Cl: confidence interval.

$. p<0.1,{ }^{*} p<0.05,{ }^{* *} p<0.01,{ }^{* \star *} p<0.001$

CS showed the highest CSS after adjusting for all the covariates in all HN SPMs (HR 0.429 compared with RS, p<0.01, Table 5) and by stages (localized SPM HR 0.430, regional SPM HR 0.249 compared with RS, $\mathrm{p}<0.05$, Table 6). Patients with localized HN SPM who received no treatment showed a comparable CSS versus RS (HR 0.685, p>0.1) (Table 6). Surgical intervention was superior compared to all the other groups in lung SPMs. However, in esophagus SPMs, no survival difference was observed among treatment types (Table 5).

\begin{tabular}{|l|l|l|l|l|}
\hline & Localized & \multicolumn{2}{l}{ Regional } \\
\hline Factors & HR & $95 \%$ Cl & HR & $95 \% \mathbf{C l}$
\end{tabular}

Gender 


\section{Cureus}

Male (R)

Female

0.609 .

$0.368-1.008$

Ethnicity

White (R)

Black

Other

Marital Status

Unmarried (R)

Married

Unknown

\% At Least Bachelors Degree

$\leq 25(\mathrm{R})$

$\square 25$

\% Families below Poverty

$>=10(R)$

1.000

$<10$

0.787

$0.418-1.480$
1.000

$1.608^{*}$

$1.052-2.457$

Index Tumor Staging

Localized (R)

Regional

Distant

Treatment

CS

RS (R)

RT

CSRT

RSRT

None
1.000

$1.944^{*}$

1.151-3.282

$3.854^{\star \star \star}$

1.774-8.374

$0.220-0.838$

$0.249^{*}$

$0.075-0.821$

1.000

1.218

0.593-2.502

$0.132-2.423$

$0.328-3.764$

$0.342-1.373$

1.000

$1.618^{\star}$

1.072-2.442

1.395

$0.432-4.511$

1.000

1.443

$0.861-2.417$

0.566

0.404

0.958-1.704

1.112

0.685
1.068

$0.595-1.918$

$2.375^{\star \star}$

\section{TABLE 6: Multivarable Analysis of Impact of Factors on HN SPM CSS by Stage}

Abbreviations: SPM, second primary cancer; CS, conservative surgery; RS, radical surgery; RT, radiation therapy; CSRT, conservative surgery with radiation; RSRT, radical surgery with radiation; HN, head and neck; R, reference; CSS; cause-specific survival; HR, hazard ratio; Cl: confidence interval. 


\section{Cureus}

$p<0.1,{ }^{\star} p<0.05,{ }^{* *} p<0.01,{ }^{* \star *} p<0.001$

\section{Discussion}

Our population-based study showed outcomes of various treatment modalities for SPMs after primary HN SCC. Based on the results of our study, it seems that aggressive surgical approaches with adjuvant radiation may be the better option compared to more conservative approaches in most patients except esophageal SPMs.

Our study (Supplementary 1) confirmed that the burden of SPMs is elevated in HNSCC patients, with a standardized incidence risk (SIR) of $1.90(\mathrm{p}<0.05)$ and an absolute excess risk (AER) of 167 per 10,000 person years (PYR) compared to the age-, gender-, and calendar year-matched normal population.

Our study found a 5-yr OS of 22.6\% from the time of diagnosis of SPM (Table 2), which is significantly lower than the recent reports on $>60 \%$-yr OS for HN cancers [16-17]. In addition, SPMs arising in the lung and esophagus had a 5 -yr OS of $12.4 \%$ and $4.9 \%$, respectively, which is significantly worse than those with an HN SPMs (Table 2) and worse than the reported corresponding primary cancer survival as well [18-19].

For HN SPMs, the management is sometimes complicated by the effects of prior treatment, including anatomical changes, risk of re-irradiation, as well as the multifocal nature of these tumors [20]. Thus, often, they are managed similarly as recurrent diseases. In our study cohort, $66.8 \%$ HN SPM patients underwent some sort of surgical resection (Table 1). The unequal survival across conservative and radical surgery groups in HN SPMs was somewhat unexpected, especially in regional SPMs. To our knowledge, there are no studies specifically comparing the outcomes between conservative and radical approaches in HN SPMs. Evidence from observational series in primary and recurrent $\mathrm{HN}$ tumors suggests that conservative procedures may be able to offer an alternative option for carefully selected patients [21-22]. For example, Ganly, et al. [22] reported that for early-stage recurrent glottis larynx tumors, partial laryngectomy gives a significantly greater 5-yr survival compared with total laryngectomy. Collectively, our findings raise important questions for surgical treatment selection in HN SPMs after HNSCC.

Interestingly, patients who did not receive any intervention for HN SPMs showed a comparable outcome to surgery (5-yr CSS 88.0\%, CSS HR 0.685, p>0.05) (Tables 3-5, Supplementary 2). We are not sure of the reason for this finding except that the retrospective nature of data limits information about histology, chemotherapy or other interventions. A possible explanation may be that 'no intervention' preserves the immunological status of the patient to fight cancer progression and such patients may be better if left untreated.

Patients with lung SPMs showed best outcomes when treated with RS alone while patients who received RT only were found to have the worst outcomes (Tables 3-5). This may be due either to advanced stage of presentation or due to conventional RT techniques in the years considered in this data collection. Conventional RT has traditionally been considered inferior to surgical resection in lung cancer treatment outcomes. However, in the last decade, increasing utilization of novel RT techniques such as stereotactic body radiation therapy (SBRT) has resulted in excellent local controls for early stage cancer, and future long term outcome data will likely result in significantly better RT outcomes, compared to conventional RT [23]. Lung SPM patients who had RSRT fared worse compared with those who underwent surgery alone (Tables 3, 5). Some studies in primary lung cancer treatment have shown that postoperative RT may not improve OS [24-25]. A randomized trial from France [26] reported that the non-cancer- 
related death increased with the RT dose delivered per fraction.

Conventional treatment options for esophagus cancer include surgical resection [27], preoperative concurrent chemoradiotherapy or neoadjuvant chemotherapy followed by resection [28-29]. Our study failed to demonstrate the superiority of any one treatment option over another in the management of esophagus SPMs.

In the diagnostic time-controlled multivariable analysis, 'SPM staging' was found to be the survival predictor among all SPMs (Table 5). Blacks did better than whites in lung SPMs (Table 5). However, we acknowledge that race was unequally distributed in our study cohort (155 blacks vs. 1497 whites), which could potentially affect statistical results. Interestingly, consistent with prior studies [30], being married was found favorable in HN and lung SPMs in univariate analysis (Supplementary 3) but not in esophagus SPMs.

As in any SEER-based study, limitations [11,14] of our study include incomplete information on adjuvant therapy, systemic treatment information, risk factors, coding reliability, and patient migration. However, we hope that the large patient numbers and the information that is available is enough to give us an idea about the management options in this subgroup of patients, and this may be useful for clinical decision making as well as generating a hypothesis for a prospective trial.

\section{Conclusions}

SPMs after an index HNSCC are considerably common and negatively impact the survival. The overall prognosis of SPMs after HNSCC has significantly improved over the years but is still poor compared with corresponding primary tumors. In HN SPMs and lung SPMs, conservative surgery with or without adjuvant radiation may be the best treatment option. Esophageal SPMs showed equivalent outcomes irrespective of the type of treatment. Some SPMs can potentially be left untreated or be managed conservatively.

\section{Appendices}




\section{Cureus}

\begin{tabular}{|c|c|c|c|c|c|}
\hline \multirow[b]{2}{*}{ Subsite } & \multirow{2}{*}{ Observed Cases } & \multirow{2}{*}{ Expected Cases } & \multicolumn{2}{|l|}{ SIR } & \multirow{2}{*}{$\begin{array}{l}\text { Excess Risk per } \\
\text { 10,000 PYR }\end{array}$} \\
\hline & & & Rate & $95 \% \mathrm{Cl}$ & \\
\hline All Sites & 20,081 & $10556 . / 1$ & $1.90^{x}$ & $1.88-1.93$ & $16 \% .31$ \\
\hline Lung and Bronchus & 5,972 & 1716.62 & $3.48^{*}$ & 3.39-3.57 & 74.75 \\
\hline Head and Neck & 4,233 & 403.55 & $10.49^{\star}$ & $10.18-10.81$ & 67.27 \\
\hline Oral Cavity and Pharynx & 3,714 & 276.47 & $13.43^{x}$ & $13.00-13.87$ & 60.39 \\
\hline Larynx & 519 & 127.08 & $4.08^{*}$ & 3.74-4.45 & 6.88 \\
\hline Esophagus & 972 & 135.58 & $7.17^{\star}$ & $6.73-7.63$ & 14.69 \\
\hline
\end{tabular}

\section{TABLE 7: Sup 1. Elevated Risk of SPM after HNSCC by Subsite}

Abbreviations: Cl: confidence interval; HNSCC, head and neck squamous cell carcinoma; PYR, persons year at risk; SIR, standardized incidence ratio; SPM, second primary cancer.

${ }^{*} \mathrm{P}<0.05$

\begin{tabular}{|c|c|c|c|c|c|c|}
\hline \multirow[b]{2}{*}{ Treatment } & \multicolumn{3}{|l|}{ All Stages } & \multicolumn{3}{|l|}{ Localized } \\
\hline & 5-yr OS (95\% Cl) & 5-yr CCS (95\% Cl) & HR & $5-y r$ OS (95\% Cl) & 5-yr CCS (95\% Cl) & HR \\
\hline & (\%) & (\%) & CSS & (\%) & (\%) & CSS \\
\hline Untreated & 47.7 (40.4-56.3) & 74.5 (67.6-82.1) & 1.217 & $59.5(50.9-69.6)$ & 88.0 (81.8-94.7) & 0.826 \\
\hline Treated & $43.2(39.6-47.1)$ & 75.8 (72.3-79.4) & 1.000 & $51.3(46.5-56.6)$ & 86.4 (82.7-90.4) & 1.000 \\
\hline
\end{tabular}

\section{TABLE 8: Sup 2. Survival Between Treated and Untreated HN SPMs}

Abbreviations: Cl: confidence interval; CSS; cause-specific survival; HN, head and neck; HR, hazard ratio; OS, overall survival; R, reference; SPM, second primary cancer.

\begin{tabular}{|l|l|l|l|}
\hline & HN & Lung & Esophagus \\
\hline Factors & HR $(95 \% \mathrm{Cl})$ & HR $(95 \% \mathrm{Cl})$ & HR $(95 \% \mathrm{Cl})$ \\
\hline $\begin{array}{l}\text { Age of Index Tumor } \\
\leq 45 \text { (referent) }\end{array}$ & 1.000 & 1.000 & \\
$46-55$ & $1.609(0.883-2.931)$ & 1.095 & 1.000 \\
$56-65$ & $2.814^{\star \star *}(1.607-4.930)$ & 1.145 & $0.805(0.419-1.546)$ \\
\hline
\end{tabular}




\section{Cureus}

66-75

$\square 75$

Age of SPM

$\leq 45$ (referent)

46-55

56-65

66-75

$\square 75$
$1.952 *(1.069-3.564)$

$1.098(0.475-2.538)$

1.000

$2.420(0.716-8.178)$

3.055 - (0.959-9.732)

$4.676^{\star \star}(1.477-14.811)$

2.777 ( $(0.858-8.992)$
1.288

$1.498^{*}$

$1.241(0.526-2.925)$

1.000

1.000

2.133 - $(0.932-4.881)$

$0.515(0.195-1.360)$

$2.135 \cdot(0.952-4.791)$

$0.684(0.274-1.706)$

2.219 . (0.991-4.967)

$0.648(0.261-1.606)$

$2.890 *(1.286-6.495)$

$0.836(0.327-2.132)$

Months since Index Tumor (Categorical)

$2-36$ (referent)
$37-72$
$73-108$
$109-144$ (>108 for Esophagus)
$145-180$
$>=181$

1.000

1.000

1.000

$0.936(0.644-1.361)$

$1.112(0.947-1.307)$

$1.524^{*}(1.043-2.228)$

$0.900(0.604-1.340)$

$1.042(0.874-1.242)$

$1.107(0.733-1.671)$

$0.929(0.589-1.466)$

$1.183 \cdot(0.969-1.445)$

$1.238(0.844-1.816)$

$0.667(0.352-1.265)$

$0.894(0.546-1.464)$

$1.121(0.886-1.420)$

$1.198 \cdot(0.990-1.450)$

Gender

Male (referent)

1.000

$0.781(0.564-1.080)$

1.000

1.000

Female

$1.092(0.962-1.240)$

$1.113(0.786-1.576)$

Ethnicity

White (referent)
Black
Other

1.000

1.000

$0.838(0.676-1.038)$

$0.956(0.705-1.298)$

1.000

$1.142(0.814-1.602)$

$0.985(0.437-2.222)$

$1.397(0.829-2.354)$

Year at diagnosis of index tumor

1973-1980 (referent)

1.000

1.000

1.000

$1981-1990$

$1.020(0.736-1.412)$

$0.943(0.773-1.152)$

$1.144(0.799-1.638)$

$0.465^{\star \star \star}(0.316-0.683)$

$0.913(0.749-1.113)$

$1.084(0.713-1.647)$

2001-2011

$0.131^{\star \star *}(0.056-0.303)$

$0.585^{\star \star \star}(0.464-0.738)$

$0.396^{* *}(0.214-0.736)$

Year at diagnosis of SPM

1973-1980 (referent) (NA for Lung)

1.000

NA

1.000

1981-1990 (referent for Lung)

1.019 (0.584-1.778)

1.000

$0.834(0.424-1.641)$

$1991-2000$

$0.814(0.470-1.409)$ 


\section{Cureus}

2001-2011

$0.248^{\star \star \star}(0.135-0.453)$

$0.789 *(0.654-0.952)$

$0.550 \cdot(0.273-1.110)$

Marital Status

Unmarried (referent)

1.000

1.000

1.000

Married

$0.709 *(0.534-0.943)$

$0.881^{*}(0.783-0.991)$

$0.808(0.604-1.081)$

Unknown

$0.774(0.439-1.365)$

$0.958(0.655-1.403)$

$0.739(0.322-1.697)$

\% At Least Bachelors Degree

$\leq 25$

$\square 25$

Median Family Income

$\leq 50$

$\square 50$

1.000

$1.249(0.919-1.696)$

1.000

$0.922(0.815-1.042)$

1.000

$1.058(0.771-1.452)$

$\%$ Families below Poverty

$>=10$ (referent)

1.000

$0.800(0.577-1.110)$

1.000

$1.025(0.883-1.191)$

1.000

1.003 (0.729-1.379)

Index Tumor Staging

Localized (referent)

Regional

Distan

1.000

$1.733^{\star \star \star}(1.304-2.302)$

$2.601^{\star * *}(1.535-4.405)$

1.000

1.096 (0.974-1.234)

$0.911(0.706-1.176)$

0.643 (0.341-1.209)

SPM Tumor Staging

Localized (referent)

Regional

Distant

1.000

$3.219^{\star \star \star}(2.378-4.358)$

$7.171^{\star \star \star}(4.738-10.855)$

Treatment

CS

$\mathrm{RS}(\mathrm{R})$

RT

CSRT

RSRT

None $0.295^{\star \star \star}(0.179-0.486)$

1.000

1.375 . (0.949-1.992)

0.364 * $(0.133-0.998)$

1.484 . (0.957-2.303)

$1.017(0.688-1.501)$
1.000

$2.262^{\star \star \star}(1.885-2.715)$

$5.869^{\star \star \star}(4.925-6.993)$

$1.146(0.815-1.610)$

$1.944^{\star \star \star}(1.361-2.777)$

NA

1.000

1.000

$3.765^{\star \star \star}(3.112-4.555)$

$1.277(0.863-1.891)$

NA

NA

$1.959^{\star * \star}(1.442-2.661)$

$1.080(0.645-1.808)$

$6.542^{\star \star \star}(5.402-7.922)$

$2.019^{* *}(1.290-3.160)$

TABLE 9: Sup 3. Univariate Analysis of Impact of Factors on CSS by Subsite 


\section{Cureus}

Abbreviations: Cl: confidence interval; CS, conservative surgery; CSS; cause-specific survival; CSRT, conservative surgery with radiation; HN, head and neck; HR, hazard ratio; OS, overall survival; R, reference; RS, radical surgery; RSRT, radical surgery with radiation; RT, radiation therapy; SPM, second primary cancer.

$. p<0.1,{ }^{*} p<0.05,{ }^{* *} p<0.01,{ }^{* *} p<0.001$

\section{Additional Information}

\section{Disclosures}

Human subjects: Consent was obtained by all participants in this study. Animal subjects: All authors have confirmed that this study did not involve animal subjects or tissue. Conflicts of interest: In compliance with the ICMJE uniform disclosure form, all authors declare the following: Payment/services info: All authors have declared that no financial support was received from any organization for the submitted work. Financial relationships: Ms. Elizabeth Mauer declare(s) a grant from Clinical and Translational Science Center at Weill Cornell Medical College (UL1-TR000457-06). Ms. Elizabeth Mauer, MS, was partially supported by the following grant: Clinical and Translational Science Center at Weill Cornell Medical College (UL1-TR000457-06). Other relationships: All authors have declared that there are no other relationships or activities that could appear to have influenced the submitted work.

\section{Acknowledgements}

Ms. Elizabeth Mauer, MS, was partially supported by the following grant: Clinical and Translational Science Center at Weill Cornell Medical College (UL1-TR000457-06).

\section{References}

1. Baxi SS, Pinheiro LC, Patil SM, et al.: Causes of death in long-term survivors of head and neck cancer. Cancer. 2014, 120:1507-1513. 10.1002/cncr.28588

2. Leon X, Del Prado Venegas M, Orus C, et al.: Metachronous second primary tumours in the aerodigestive tract in patients with early stage head and neck squamous cell carcinomas. Eur Arch Otorhinolaryngol. 2005, 262:905-909. 10.1007/s00405-005-0922-5

3. Slaughter DP, Southwick HW, Smejkal W: Filed cancerization' in oral stratified squamous epithelium. Clinical implications of multicentric origin. Cancer. 1953, 6:963-968. 10.1002/1097-0142(195309)6:5<963::AID-CNCR2820060515>3.0.CO;2-Q

4. Braakhuis BJ, Tabor MP, Leemans CR, et al.: Second primary tumors and field cancerization in oral and oropharyngeal cancer: molecular techniques provide new insights and definitions. Head Neck. 2002, 24:198-206. 10.1002/hed.10042

5. Chuang SC, Scelo G, Tonita JM, et al.: Risk of second primary cancer among patients with head and neck cancers: a pooled analysis of 13 cancer registries. Int J Cancer. 2008, 123:23902396. 10.1002/ijc.23798

6. Peck BW, Dahlstrom KR, Gan SJ, et al.: Low risk of second primary malignancies among never smokers with human papillomavirus-associated index oropharyngeal cancers. Head Neck. 2013, 35:794-799. 10.1002/hed.23033

7. Leon X, Quer M, Diez S, et al.: Second neoplasm in patients with head and neck cancer . Head Neck. 1999, 21:204-210.

8. Morris LG, Sikora AG, Patel SG, et al.: Second primary cancers after an index head and neck cancer: subsite-specific trends in the era of human papillomavirus-associated oropharyngeal cancer. J Clin Oncol. 2011, 29:739-746. 10.1200/JCO.2010.31.8311

9. Chen MC, Huang WC, Chan CH, et al.: Impact of second primary esophageal or lung cancer on survival of patients with head and neck cancer. Oral Oncol. 2010, 46:249-254.

10.1016/j.oraloncology.2010.01.002

10. Jayaprakash V, Cheng C, Reid M, et al.: Previous head and neck cancers portend poor prognoses in lung cancer patients. Ann Thorac Surg. 2011, 92:1056-1060. 


\subsection{6/j.athoracsur.2011.03.146}

11. Yu JB, Gross CP, Wilson LD, et al.: NCI SEER public-use data: applications and limitations in oncology research. Oncology (Williston Park). 2009, 23:288-295.

12. Multiple Primary - Standardized Incidence/Mortality Ratios (MP-SIR/SMR) . Accessed: April 23, 2017: https://seer.cancer.gov/seerstat/mp-sir.html.

13. Lim MC, Won YJ, Lim J, et al.: Second primary cancer after diagnosis and treatment of cervical cancer. Cancer Res Treat. 2016, 48:641-649. 10.4143/crt.2014.326

14. Jain KS, Sikora AG, Baxi SS, et al.: Synchronous cancers in patients with head and neck cancer: risks in the era of human papillomavirus-associated oropharyngeal cancer. Cancer. 2013, 119:1832-1837. 10.1002/cncr.27988

15. Curtis RE, Freedman DM, Ron E, et al.: New malignancies among cancer survivors: SEER Cancer Registries, 1973-2000. National Cancer Institute. 2006, NIH Publ. No. 055032:Accessed: May 26, 2017: https://seer.cancer.gov/archive/publications/mpmono/MPMonograph_complete.pdf .

16. SEER stat fact sheets: oral cavity and pharynx cancer . (2006-2012). Accessed: May 26, 2017 : https://seer.cancer.gov/statfacts/html/oralcav.html .

17. SEER stat fact sheet: larynx cancer . (2006-2012). Accessed: May 26, 2017: https://seer.cancer.gov/statfacts/html/laryn.html.

18. SEER stat fact sheets: lung and bronchus cancer . (2006-2012). Accessed: May 26, 2017: http://seer.cancer.gov/statfacts/html/lungb.html.

19. SEER stat fact sheets: esophageal cancer . (2006-2012). Accessed: May 26, 2017 : https://seer.cancer.gov/statfacts/html/esoph.html.

20. Ho AS, Kraus DH, Ganly I, et al.: Decision making in the management of recurrent head and neck cancer. Head Neck. 2014, 36:144-151. 10.1002/hed.23227

21. Misono S, Marmor S, Yueh B, et al.: Treatment and survival in 10,429 patients with localized laryngeal cancer: a population-based analysis. Cancer. 2014, 120:1810-1817. $10.1002 /$ cncr.28608

22. Ganly I, Patel SG, Matsuo J, et al.: Results of surgical salvage after failure of definitive radiation therapy for early-stage squamous cell carcinoma of the glottic larynx. Arch Otolaryngol Head Neck Surg. 2006, 132:59-66. 10.1001/archotol.132.1.59

23. Parashar B, Port J, Arora S, et al.: Analysis of stereotactic radiation vs. wedge resection vs. wedge resection plus Cesium-131 brachytherapy in early stage lung cancer. Brachytherapy. 2015, 14:648-654. 10.1016/j.brachy.2015.04.001

24. Lally BE, Zelterman D, Colasanto JM, et al.: Postoperative radiotherapy for stage II or III nonsmall-cell lung cancer using the surveillance, epidemiology, and end results database. J Clin Oncol. 2006, 24:2998-3006. 10.1200/JCO.2005.04.6110

25. PORT Meta-analysis Trialists Group: Postoperative radiotherapy in non-small-cell lung cancer: systematic review and meta-analysis of individual patient data from nine randomised controlled trials. Lancet. 1998, 352:257-263. 10.1016/S0140-6736(98)06341-7

26. Dautzenberg B, Arriagada R, Chammard AB, et al.: A controlled study of postoperative radiotherapy for patients with completely resected nonsmall cell lung carcinoma. Cancers. 1999, 86:265-273. 10.1002/(SICI)1097-0142(19990715)86:2<265::AID-CNCR10>3.0.CO;2-B

27. Ajani JA, Barthel JS, Bentrem DJ, et al.: Esophageal and esophagogastric junction cancers: clinical practice guidelines in oncology. J Natl Compr Canc Netw. 2011, 9:830-887. Accessed: May 26, 2017: http://www.jnccn.org/content/9/8/830.full.pdf.

28. van Hagen P, Hulshof MC, van Lanschot JJ, et al.: Preoperative chemoradiotherapy for esophageal or junctional cancer. N Engl J Med. 2012, 366:2074-2084.

10.1056/NEJMoa1112088

29. Tepper J, Krasna MJ, Niedzwiecki D, et al.: Phase III trial of trimodality therapy with cisplatin, fluorouracil, radiotherapy, and surgery compared with surgery alone for esophageal cancer: CALGB 9781. J Clin Oncol. 2008, 26:1086-1092. 10.1200/JCO.2007.12.9593

30. Aizer AA, Chen MH, McCarthy EP, et al.: Marital status and survival in patients with cancer . J Clin Oncol. 2013, 31:3869-3876. 10.1200/JCO.2013.49.6489 